
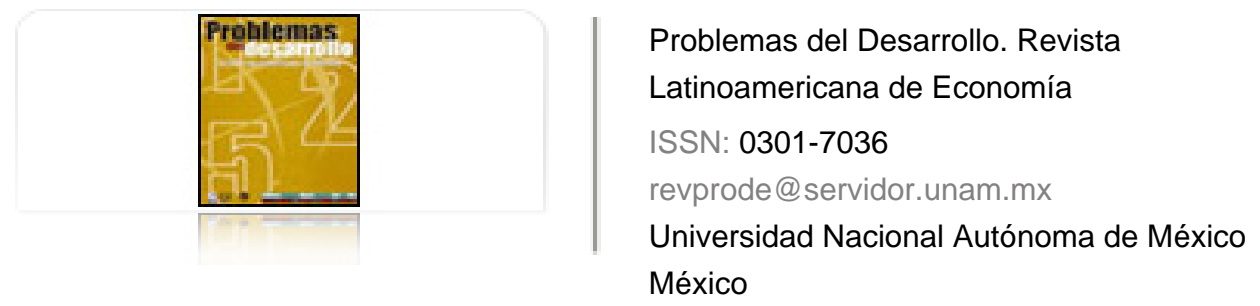

Sobarzo Fimbres, Horacio Enrique

Relaciones fiscales intergubernamentales en México: evolución reciente y perspectivas Problemas del Desarrollo. Revista Latinoamericana de Economía, vol. 40, núm. 156, enero-marzo, 2009, pp. 11-28

Universidad Nacional Autónoma de México

Distrito Federal, México

Disponible en: http://www.redalyc.org/articulo.oa?id=11820096009

- Cómo citar el artículo

- Número completo

- Más información del artículo

Página de la revista en redalyc.org

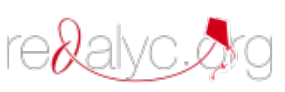

Sistema de Información Científica

Red de Revistas Científicas de América Latina, el Caribe, España y Portugal Proyecto académico sin fines de lucro, desarrollado bajo la iniciativa de acceso abierto 


\title{
RELACIONES FISCALES INTERGUBERNAMENTALES EN MÉXICO: EVOLUCIÓN RECIENTE Y PERSPECTIVAS
}

\author{
Horacio Enrique Sobarzo Fimbres*
}

Fecha de recepción: 20 de agosto de 2008. Fecha de aceptación: 4 de diciembre de 2008.

\section{Resumen}

El trabajo intenta caracterizar la situación actual y delinear alguna perspectiva sobre el estado de las relaciones fiscales intergubernamentales en México, sobre todo la relación entre gobierno federal y gobiernos estatales. Para tal efecto se describe la evolución de las relaciones entre estos ámbitos de gobierno en las últimas tres décadas, distinguiendo ingresos y gastos. Una segunda parte del trabajo trata de introducir los elementos teóricos fundamentales que puedan ser útiles para entender el estado actual de estas relaciones en México y su posible evolución en el futuro; se trata además de identificar las tendencias y los riesgos. La conclusión central es que se requiere de una mayor participación de los gobiernos estatales en el proceso de recaudación de impuestos y una redefinición del papel del gobierno federal.

Palabras clave: federalismo fiscal, relaciones fiscales intergubernamentales, recaudación de impuestos, gasto público y coordinación fiscal.

* Profesor-investigador del Centro de Estudios Económicos de El Colegio de México. Correo electrónico: hsobarzo@colmex.mx.

Una versión previa de este trabajo apareció publicada en el volumen Federalismo y Descentralización, editado por CONAGO, 2008. La presente versión, a diferencia de la referida, incorpora una discusión sobre la teoría existente respecto a los beneficios y problemas potenciales de la competencia tributaria. 
Abstract

The study aims to characterize the present situation and to delineate some perspective on the state of inter-governmental fiscal relations in Mexico, above all on the relationship between the federal government and the state governments. For this purpose, we describe the evolution of relations between these government areas over the last three decades, distinguishing incomes and spending. A second part of the study aims to introduce the fundamental theoretical elements that could be useful for understanding the present state of these relationships in Mexico and their possible evolution in the future; it also tries to identify the trends and risks. The central conclusion is that it would require a greater participation of the state governments in the process of tax collection and a redefinition of the role of the federal government.

Key words: fiscal federalism, inter-governmental fiscal relations, tax collection, public spending and fiscal coordination.

\section{Résumé}

Ce travail a pour but de caractériser la situation actuelle et délimiter une perspective de l'état des relations fiscales entre gouvernements au Mexique, surtout la relation entre le gouvernement fédéral et les gouvenements des états fédérés. À cet effet, il y est décrit l'évolution des relations entre ces niveaux de gouvernement au cours des trois dernières décennies, en distinguant revenus et dépenses. Une seconde partie du travail tente d'introduire les éléments théoriques fondamentaux qui peuvent être utiles pour comprendre l'état actuel de ces relations au Mexique et leur possible évolution dans le

futur; il s'agit en outre d'identifier les tendances et les risques. La conclusion centrale est qu'il faut une plus grande participation des états fédérés dans le processus de recouvrement des impôts et une redéfinition du rôle du gouvernement fédéral.

Mots clés: fédéralisme fiscal, relations fiscales intergouvernementales, recouvrement des impôts, dépense publique et coordination fiscale.

\section{Resumo}

O trabalho tenta caracterizar a situação atual e indicar alguma perspectiva sobre o estado das relações fiscais intergovernamentais no México, sobre tudo a relação entre governo federal e governos estaduais. Para tal efeito se descreve a evolução das reações entre estes âmbitos de governo nas três ultimas décadas, distinguindo ingressos e gastos. Uma segunda parte do trabalho trata de introduzir os elementos teóricos fundamentais que possam ser úteis para entendermos o estado atual dessas relações no México e sua possível evolução no futuro; trata-se, além disso, de identificar as tendências e os riscos. A conclusão central é que se requere de uma maior participação dos governos estaduais no processo de arrecadação de impostos e uma redefinição do papel do governo federal.

Palavras chave: Federalismo fiscal, relações intergovernamentais, arrecadação de impostos, gastos públicos e coordenação fiscal.

\section{Desarrollo}




\section{Introducción}

partir de la introducción del Impuesto al Valor Agregado (IVA) en 1980, las
entidades federativas cedieron el grueso de su capacidad recaudatoria al go-
bierno federal y en el seno del llamado Sistema Nacional de Coordinación Fiscal (SNCF) se diseñó un sistema de transferencias (participaciones) que hasta la fecha subsiste. Las reformas de 1980 simplificaron el sistema tributario en su conjunto $\mathrm{y}$ - a la postre, a pesar del pobre desempeño tributario del país — redundaron en mayores ingresos, particularmente para el ámbito estatal de gobierno.

A pesar de que con el tiempo se modificaron los criterios de reparto de las participaciones, la esencia del sistema se mantuvo por el lado del ingreso, pues desde entonces las capacidades recaudatorias se han mantenido en el gobierno federal. Sin embargo, por el lado del gasto —en el contexto de una creciente apertura económica y política - se inició un importante proceso de descentralización, mismo que arrancara en 1992 con la llamada descentralización educativa y que después habría de extenderse a otras áreas. En la actualidad, por cada peso de gasto en el sector público más de la mitad lo llevan a cabo gobiernos estatales y municipales.

Sin embargo, el proceso no necesariamente ha conducido a una mayor autonomía de los gobiernos subnacionales, entre otras cosas porque buena parte del gasto descentralizado a los estados es condicionado y en algunas áreas importantes las directrices y políticas, en esencia, han permanecido en el ámbito federal. Más aún, es difícil generalizar porque el proceso ha sido distinto por áreas y por regiones, lo cual no es sorprendente dada la gran heterogeneidad territorial del país. A lo anterior habría que añadir que la transparencia en el ejercicio del gasto estatal y los correspondientes sistemas de rendición de cuentas han avanzado relativamente poco, en comparación con el ámbito federal de gobierno, y de manera más heterogénea entre entidades federativas.

El argumento central de este documento es que si se continúa esta tendencia de descentralización del gasto a las entidades federativas sin descentralizar también la recaudación —aunque sea de manera parcial—, se pone en riesgo la solvencia del sistema fiscal en su conjunto, pues en caso de problemas financieros originados en los gobiernos subnacionales, el gobierno federal es a final de cuentas el garante de última instancia. En el mediano y largo plazos la única forma de disminuir este riesgo es involucrando a las entidades federativas en la recaudación en un grado mucho mayor que el que ahora prevalece. Si en efecto los gobiernos subnacionales, particularmente los estatales, se involucran más en la recaudación (parcial y coordinada) de impuestos de base amplia, es de esperarse que no sólo se reduzca el riesgo de la pérdida de 
solvencia, sino también que en sí mismo ello induzca una mayor responsabilidad fiscal en el ejercicio del gasto (para una estimación del potencial recaudatorio de los estados en impuestos de base amplia véase Sobarzo, 2006).

La estructura del documento es la siguiente: en primera lugar, se revisan los antecedentes y la evolución reciente de los ingresos y los gastos en el contexto del federalismo fiscal y se trata de caracterizarlos brevemente. Luego se presenta un resumen de los elementos teóricos fundamentales acerca de las ventajas y las desventajas de la competencia tributaria, a fin de arrojar un poco de luz respecto al planteamiento central del presente trabajo, a saber, que se requiere aumentar la capacidad recaudatoria de los gobiernos estatales. En seguida se hacen algunos comentarios sobre la necesidad de estudiar con más detalle los determinantes del gasto en los estados. Finalmente, se presenta una reflexión sobre los problemas potenciales del federalismo fiscal en México.

\section{Antecedentes y evolución de las relaciones fiscales intergubernamentales en México}

\section{Evolución de los ingresos públicos}

Si bien la historia de las relaciones fiscales intergubernamentales puede ubicarse en el surgimiento de las convenciones nacionales fiscales, en los años 1925, 1943 y 1947, es a partir de 1980, con la introducción del IVA, cuando se crea lo que es hoy el SNCF, y se le da forma al actual sistema.

Los cambios ocurridos en 1980 pueden quizás caracterizarse como una reforma fiscal exitosa, no sólo porque se modificó de fondo el sistema de relaciones fiscales intergubernamentales, sino porque también se simplificó el sistema tributario en su conjunto, pues se eliminó una serie de impuestos especiales que implicaban la existencia de tributación múltiple. ${ }^{1}$ Sin duda, la modificación más trascendental fue que con la introducción del IVA, a cargo del gobierno federal, los estados prácticamente cedieron al gobierno central la función recaudatoria de su fuente principal de ingresos, que era su participación en el llamado Impuesto Sobre Ingresos Mercantiles (ISIM). Debe recordarse que aunque el IVA se introdujo como un impuesto federal, después de 1980 las entidades continuaron desempeñando una función recaudatoria,

1 De hecho, con la nueva tasa del IVA se calculó que debería ser suficiente para compensar la recaudación suspendida del Impuesto Sobre Ingresos Mercantiles (4\%), un impuesto de 30\% al consumo suntuario, 25 impuestos federales y 300 estatales (Gil y Thirsk, 2005). 
es decir, siguieron participando en la administración del impuesto, en el entendido de que los ingresos recaudados eran del gobierno federal que, a su vez, otorgaría a las entidades al menos los ingresos suspendidos antes del cambio en 1980.

En un periodo de abundancia petrolera esta modificación fue posible no sólo por el fuerte centralismo y control político en la Presidencia de la República, sino también porque la bonanza petrolera permitió que bajo el nuevo sistema de participaciones ninguna entidad empeorara en términos de los ingresos recibidos. De hecho, todas tuvieron una mejoría.

En su origen, el sistema de participaciones tuvo un carácter fundamentalmente compensatorio, es decir, las participaciones a los estados se distribuían con el criterio de resarcir a los estados por los ingresos que obtenían en el sistema anterior.

Con el paso de los años el sistema de distribución de participaciones sufrió modificaciones en los criterios de reparto. Hasta 2007 el Fondo General de Participaciones — con mucho el más importante del total de participaciones a los estados— se distribuyó bajo los siguientes tres criterios hasta antes de la reforma fiscal de 2008: 45.17\% con base en el número de habitantes, $45.17 \%$ con base en los llamados impuestos asignables territorialmente, y el restante $9.66 \%$ en función inversa a los dos criterios anteriores. ${ }^{2}$ A partir de 2008, como resultado de la reforma fiscal, los incrementos se rigen de manera adicional por crecimiento del Producto Interno Bruto (PIB) y recaudación de ingresos propios, si bien ponderados por el peso poblacional de cada entidad federativa.

En buena medida, las modificaciones a los criterios de reparto han obedecido a ajustes impulsados por las entidades federativas, pero más allá de si el esquema o los criterios del reparto son "justos", lo cierto es que en mayor o menor grado todos los estados han visto un crecimiento importante de sus ingresos por este concepto. Así, en el periodo 1980-2005 el crecimiento estatal real acumulado por este concepto fue de $155.7 \%$, muy superior al crecimiento del PIB — que fue de $85.1 \%$ - o de la propia Recaudación Federal Participable (RFP) — que en el mismo periodo creció en términos reales 78.4\%—. En términos per capita, a pesos de 1993, las participaciones promedio en el ámbito nacional pasaron de 386.3 a 552.1 pesos en 2005 (UCEF, 2006).

2 El monto total a distribuir en el Fondo General de Participaciones es un porcentaje (20\%) de lo que se conoce como Recaudación Federal Participable (RFP), que se integra por todos los impuestos federales y los derechos sobre extracción de petróleo y minería, además de los recursos, ajustados por devoluciones, compensaciones, participaciones específicas en IEPS e incentivos autoliquidables, tenencia, ISAN, y colaboración administrativa. 
Gráfica 1

Relación de participaciones-PIB estatal 1995-2002

pesos constantes

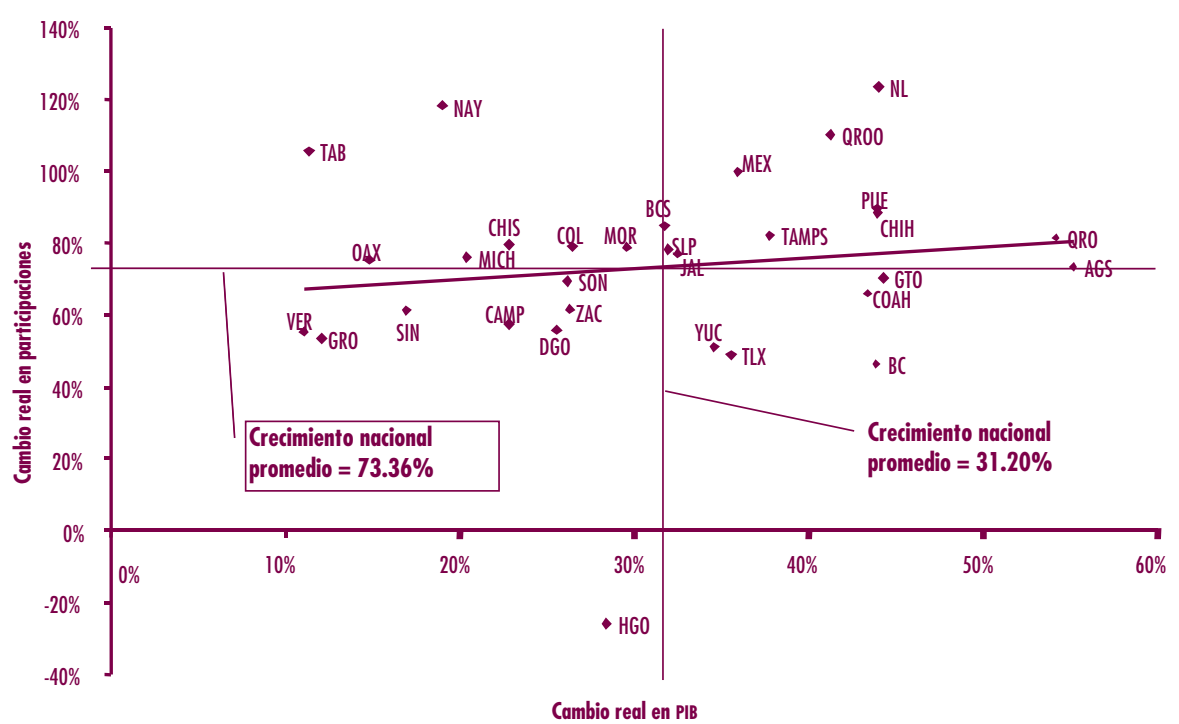

Ahora bien, este incremento en las participaciones no ha sido homogéneo entre entidades y no es sencillo establecer algún patrón de comportamiento. Por ejemplo, no se le ha relacionado con el crecimiento del PIB en los estados. En la gráfica 1 se puede apreciar cómo hay estados que aun cuando han aumentado sus participaciones por arriba de la media nacional, su PIB decreció y viceversa. Si bien el PIB no es el único indicador a tomar en cuenta para evaluar los efectos de las participaciones, puede reconocerse que hace falta analizar a fondo la relación entre los objetivos de distribución de participaciones y los criterios con que éstos se asignan. Autores como Cabrero (1995) señalan que la creciente dependencia de participaciones federales ha exacerbado las disparidades regionales y no ha permitido la aparición de iniciativas regionales dirigidas a mejorar el desarrollo regional.

En resumen, la reforma de 1980, si bien permitió simplificar el sistema impositivo, implicó que en lo sucesivo el gobierno federal sería el encargado de recaudar los impuestos de base amplia, ${ }^{3}$ lo que implicó que las entidades federativas cedieran al gobierno federal la función recaudatoria. Este sistema ha propiciado un aumento

3 Impuesto Sobre la Renta (ISR), Impuesto al Valor Agregado (IVA) y Impuesto Especial a la Producción y Servicios (IEPS). 


\section{Gráfica 2}

Ingresos de los estados

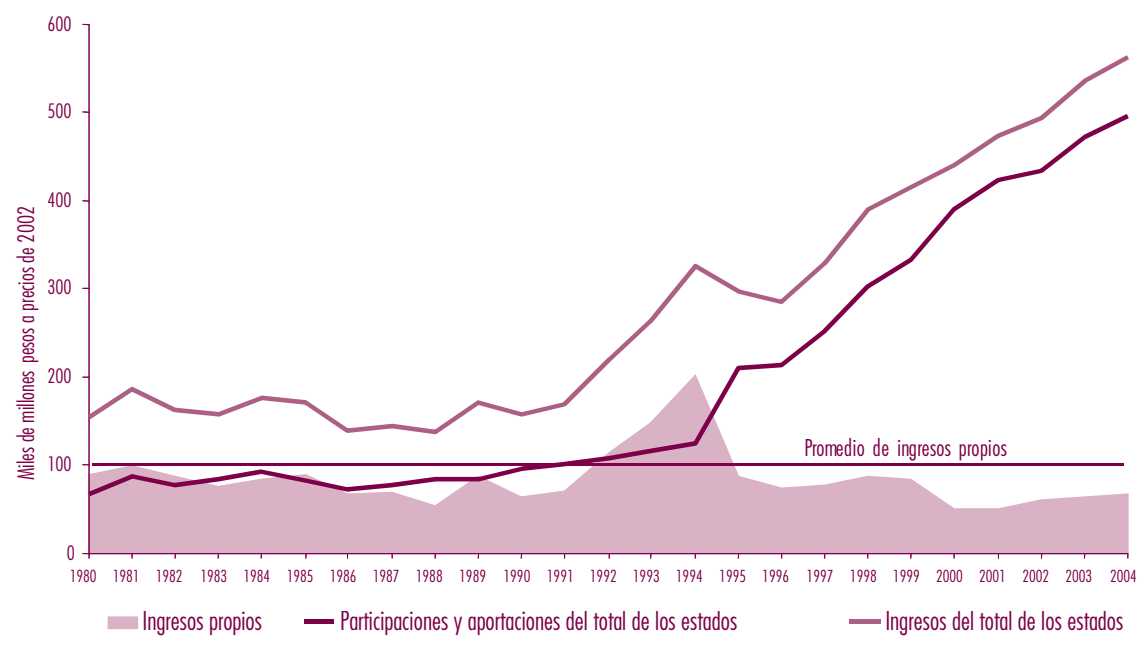

Fuente: INEGI, Finanzas Públicas Estatales y Municipales de México. Sistema de información energética. Dirección General Adjunta de Estadística de la Hacienda Pública, XAB21 - Ingresos petroleros.

importante de ingresos a los estados. Como se verá más adelante, ello ha implicado que el sistema resultante se caracterice por una fuerte concentración recaudatoria en el gobierno federal. En el año 2005, del total de ingresos de los estados, en promedio, 92.4\% proviene de transferencias federales, es decir, sólo 7.6\% de los ingresos totales fueron ingresos propios. ${ }^{4}$

\section{Evolución del gasto público estatal}

Por el lado de los ingresos, quizá la característica más destacable sea que existe, como ya se comentó, una fórmula de distribución de participaciones, y aunque ha sido modificada más de una vez en los últimos 25 años, los criterios con que se asignan los recursos siempre han sido claros, más allá de sí han sido "justos".

4 Ciertamente hay diferencias de comportamiento individual. Por ejemplo, en 2005 los ingresos propios de Chihuahua y Nuevo León fueron de $15.3 \%$ y $12.8 \%$ respectivamente, en tanto que estados como Tlaxcala y Puebla registraron porcentajes de 3.5\% cada uno. De entre los impuestos estatales más importantes destacan el impuesto sobre nómina (30 estados y el DF) y hospedaje (29 estados y el DF). 
Gráfica 3

gasto por nivel de gobierno

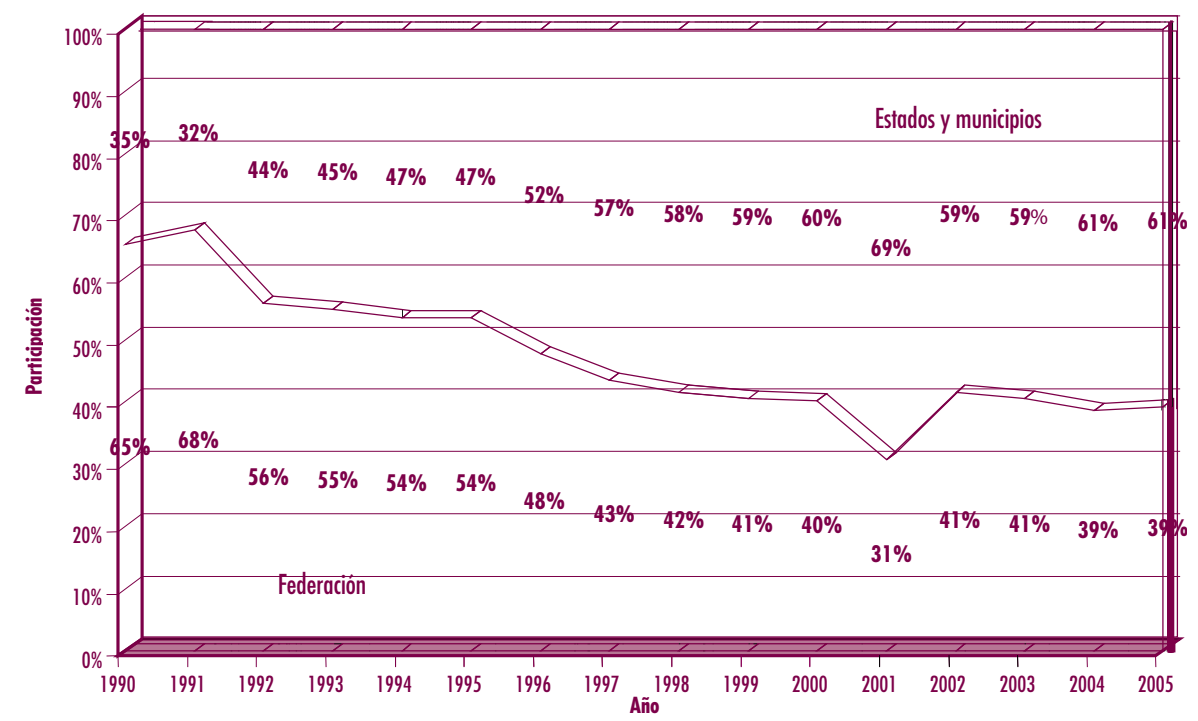

Fuente: SHCP, Diagnóstico Integral de la Situación Actual de las Hacinedas Püblicas Estatales y Municipales [en línea]. Unidad de Coordinación con Entidades Federativas, Mayo de 2006, p.12 [ref. 21 de junio de 2006]. Disponible en Internet en: <http://www.apartados.hacienda.gob.mx/ucef/ index_documentos_relevantes.html>

La evolución de los gastos ha sido diferente. Se pueden distinguir con claridad dos etapas. La primera comprende el periodo anterior a 1992, que se caracteriza por una fuerte centralización de gastos en el ámbito federal, pero sobre todo por el alto grado de discrecionalidad en su asignación entre entidades federativas. Si bien la distribución de recursos en la década de los ochenta se regía por los planes de desarrollo, en la práctica no existían criterios objetivos de reparto y, en buena medida, la asignación se definía por las decisiones del Ejecutivo y su relación con los gobernadores. ${ }^{5}$

En la década de los noventa, en gran medida motivado por las trasformaciones políticas y económicas del país, este escenario comienza a modificarse de manera significativa a partir del proceso de descentralización educativa iniciado en 1992 y al que siguieron algunas otras áreas como salud y gasto social, destacando más recientemente la conformación del llamado Ramo 33 y el Programa de Aportaciones para el Fortalecimiento de las Entidades Federativas (PAFEF).

5 De hecho se ha documentado que el propio calendario electoral era un determinante importante (véase Molinar y Weldon, 1994). 
Como resultado de este proceso, de acuerdo con información de la Secretaría de Hacienda y Crédito Público (SHCP), en la actualidad las entidades federativas reciben transferencias condicionadas y participaciones (transferencias no condicionadas), lo que hace que los estados y municipios ejerzan $61 \%$ del gasto total gubernamental frente a 39\% del gobierno federal (véase gráfica 3).

La característica más importante de estos procesos es que - a diferencia de las décadas anteriores a los noventa - la discrecionalidad con la que se asignaban los gastos en las entidades prácticamente ha desaparecido, ya que en todos los casos existen fórmulas de reparto, o bien — como sucede con el gasto educativo— su asignación obedece a la inercia proveniente al inicio del proceso. En este sentido, puede hablarse de un gran avance en relación con los años anteriores, pues hoy existe más certeza y certidumbre en el ejercicio del gasto estatal.

Dicho lo anterior, es importante resaltar que estos procesos de descentralización no han estado exentos de problemas ya que en muchos casos los procesos de homologación generaron altos costos en algunos estados, debido a que la diversidad en la calidad y cantidad de servicios estatales era muy amplia. Destacan los casos de educación y, en menor medida, de salud, pues la existencia de servicios educativos estatales previos a la descentralización terminó penalizando a aquellas entidades que habían desarrollado sus propios sistemas educativos. Es decir, los estados que habían desarrollado sistemas educativos estatales propios tuvieron que pagar altos costos para homologar los servicios con el sistema federal, en tanto que aquellas entidades que nunca hicieron esfuerzos por desarrollar un sistema educativo estatal terminaron enfrentando costos relativamente menores. Esta situación se vio exacerbada por el hecho de que el Sindicato Nacional de Trabajadores de la Educación (SNTE) se mantuvo como una entidad nacional.

Quizás es necesario dimensionar de forma adecuada este importante proceso de descentralización, pues si bien las entidades ejercen hoy mayores recursos que la Federación, en cierto sentido el cambio operado fue de naturaleza puramente contable, toda vez que los recursos que antes gastaba la Federación en los estados se transfirieron a éstos para que continuaran ejerciéndolos en las mismas áreas y casi con los mismos criterios. Dada la naturaleza condicionada de estas transferencias, no se modificó de manera sustancial el grado de libertad de las entidades para ejercer recursos propios. Es difícil realizar una evaluación del proceso de descentralización por la diversidad de resultados, tanto por área como por sus impactos territoriales. Sin embargo, podría afirmarse que en educación, por ejemplo, no se han logrado transferir directrices y políticas. Incluso, en general, un aspecto fallido del proceso es 
que no se ha logrado que los estados aporten recursos propios, como se proponía en el acuerdo original. En materia de salud hay un escenario de resultados diversos, dependiendo del estado al que se haga referencia pero se ha avanzado relativamente poco en la mejora de la calidad del servicio a la población abierta, así como en la cobertura, sobre todo en las zonas más marginadas del país, donde se esperaba que por la mayor cercanía de los gobiernos estatales a la población ésta pudiera ampliarse. Algo similar puede afirmarse en las áreas de desarrollo social y seguridad pública. Sin embargo, esta evaluación debe tener la atenuante — si así pudiera llamársele — de que durante el periodo de referencia el desempeño económico global del país ha sido pobre, por lo que sería injusto atribuir los resultados sólo al proceso de descentralización.

\section{Excedentes petroleros}

Para concluir con esta sección de gasto, vale la pena destacar que en los últimos años, en particular a partir de 2000, se ha registrado un crecimiento importante en los excedentes petroleros, propiciado por un gran incremento de su precio en el mercado mundial. A partir de 1998 - cuando el precio promedio del barril de la mezcla mexicana tuvo su nivel más bajo, por debajo de los 10 dólares— se ha incrementado su precio de forma permamente y significativa. ${ }^{6}$ Entre los grandes beneficiarios de estos excedentes se encuentran las entidades federativas, ya que a partir de 2003 se les destina 50\% de los excedentes, vía del llamado Fideicomiso para la Infraestructura en los Estados (FIES), en tanto que el restante 50\% se destinó a gastos de inversión de PEMEX.7 $\mathrm{La}$ correlación entre ingresos totales de los estados y el precio internacional de la mezcla mexicana de petróleo se hizo particularmente fuerte en el periodo 1999-2004.

Este proceso, aunque parece positivo porque ha significado más recursos a las entidades federativas, tiene el problema de que la transparencia y rendición de cuentas en materia de gasto en el plano estatal no ha avanzado, en promedio, con la misma rapidez que en el ámbito federal y, de hecho, entre las propias entidades federativas existe un escenario de una gran heterogeneidad. De manera adicional, en el plano de las finanzas públicas nacionales se corre el riesgo de que, como sucediera con la bonanza petrolera de finales de los setenta, los esfuerzos de reforma fiscal se pospon-

6 Para el mes de agosto de 2008 el precio del barril mexicano llegó a estar por encima de los 120 dólares.

7 Para 1995 se definió como excedentes petroleros a los recursos generados cuando el precio de la mezcla mexicana del petróleo superara los 27 dólares por barril. 
gan una vez más. Cabe resaltar que la más reciente reforma fiscal, vigente a partir de 2008, ha sido un intento por reducir la dependencia de las finanzas públicas de los ingresos asociados a la actividad petrolera.

En suma, podría decirse que en el ámbito de las relaciones fiscales intergubernamentales ha habido un avance significativo en México. A diferencia de los años ochenta y décadas anteriores, la discrecionalidad en la asignación del gasto entre entidades prácticamente ha desaparecido y, en consecuencia, hoy existe mayor certidumbre y certeza en la planeación del gasto estatal.

Ahora bien, a pesar de que hoy las entidades y municipios ejercen $61 \%$ del gasto frente a $39 \%$ del gobierno federal, el grueso del gasto descentralizado es de tipo condicional, por lo que la independencia en el gasto estatal no es tan alta como en principio los números parecen sugerir.

Sin embargo, hay que reconocer que la evolución del SNCF desde 1980 a la fecha ha incrementado significativamente los recursos dirigidos a los estados. Si a ello se suman los excedentes petroleros de los últimos años y las llamadas aportaciones, resulta que en efecto los estados se encuentran entre los principales beneficiarios no sólo de la abundancia petrolera, sino del proceso de descentralización. Al igual que sucede con la evolución de las participaciones, el reparto de recursos federales condicionados no parece obedecer a algún criterio en particular; más bien ha sido el resultado de circunstancias históricas o de consideraciones de tipo político. Así, por ejemplo, los excedentes petroleros distribuidos a las entidades federativas, una vez determinado el monto (50\%), se han distribuido con base en los coeficientes del Fondo General de Participaciones (FGP), cuyo comportamiento, como ya se comentó, no es sencillo establecer. En otras palabras, la evolución de los recursos distribuidos a las entidades parece haber obedecido en buena medida a circunstancias históricopolíticas, y no necesariamente vinculadas a criterios antes definidos.

Por el lado del ingreso, debe destacarse que subsiste una centralización muy fuerte en el ámbito federal, lo que - aunado a la fuerte descentralización del gasto — plantea interrogantes y problemas potenciales que habrán de discutirse más adelante.

\section{Contexto teórico y características del sistema actual y problemas presentes y potenciales}

\section{Problema general y contexto teórico}

El resultado del proceso descrito a muy grandes rasgos en la sección anterior es un sistema fuertemente centralizado en el gobierno federal en lo referente a recaudación 
y, por el lado del gasto, con un grado importante de descentralización a los gobiernos subnacionales. Es decir, existe lo que se le ha dado en llamar un muy marcado desequilibrio vertical entre los ámbitos estatal y federal de gobierno.

Desde el punto de vista de la eficiencia económica, un sistema en el que la recaudación se concentra en el gobierno federal, acompañado de un apropiado sistema de transferencias a los gobiernos subnacionales, puede ser visto como adecuado (Gordon, 1983). Sin embargo, análisis más recientes han puesto énfasis en el hecho de que un sistema de estas características puede dar origen a lo que se ha llamado restricciones presupuestales laxas, es decir, en ciertas circunstancias este esquema de recaudación centralizada induce a los gobiernos subnacionales a una expansión excesiva del gasto que, eventualmente, habrá de ser solventada con recursos adicionales provenientes del gobierno central. En esencia, se argumenta que los presupuestos de los gobiernos estatales tienden a crecer de manera excesiva por la poca vinculación entre el gasto (proveniente de transferencias) y los reducidos ingresos propios. Como se comenta más adelante, cuando se hace referencia al llamado efecto matamoscas, ello puede suceder por diversas razones, entre éstas aspectos políticos, poca identificación frente a la ciudadanía local e, incluso, ilusión monetaria. Por ejemplo, de acuerdo con Rodden, Gunnar y Litvack (2003), cuando los gobiernos subnacionales disponen de pocas facultades recaudatorias de forma tal que son muy dependientes de las transferencias federales, claramente tienen muy poca flexibilidad para enfrentar choques o situaciones adversas, por lo que en periodos de crisis fiscales la presión recaerá en el gobierno central. Como los gobiernos subnacionales conocen esta situación, tienen poca motivación o incentivos para ser fiscalmente responsables, pues existe la expectativa de que de manera eventual habrán de ser "rescatados" por el gobierno federal.

De forma adicional al desbalance vertical mencionado líneas atrás, otra circunstancia que puede dar origen a restricciones fiscales laxas puede presentarse en caso de que los gobiernos subnacionales no atiendan con suficiencia algunos servicios que puedan trascender a la localidad, como es el caso de las pensiones. En circunstancias como éstas los gobiernos centrales eventualmente se pueden ver obligados a atender la demanda. En general, tanto la estructura fiscal como las instituciones políticas pueden influir en la mayor o menor proclividad de parte de los gobiernos centrales para fungir como garantes de última instancia a comportamientos fiscalmente irresponsables de los gobiernos subnacionales. Aquí la razón en el crecimiento excesivo del gasto de los gobiernos subancionales proviene de la existencia de externalidades que de manera clara trascienden a la localidad. Por ejemplo, la certeza de que el go- 
bierno central de manera eventual tendrá que hacer frente al problema de pensiones (nacional) explica el porqué los estados pueden hacer crecer el gasto corriente sin que sea necesario atender el problema de su financiamiento.

De hecho, en un esquema centralizado y donde los gobiernos subnacionales tienen poca autonomía tributaria o de ingresos, los actores políticos en los gobiernos subnacionales no serán necesariamente los responsables frente a los ciudadanos. Se espera que si las transferencias (o parte de ellas) están muy influenciadas por consideraciones políticas, entonces es difícil mantener la credibilidad de que no habrá rescate del gobierno central. Wildasin (1997) argumenta que, al revés de lo que se piensa, los problemas de disciplina fiscal se presentan no porque haya mucha descentralización fiscal sino porque, contrariamente, hay muy poca, haciendo referencia, por supuesto, a la incipiente capacidad recaudatoria de los gobiernos subnacionales.

Las experiencias recientes en los últimos años, por ejemplo en Argentina, resaltan la necesidad de diseñar un sistema que permita (obligue) a los estados, si bien de forma parcial, a involucrarse en el proceso recaudatorio (véase Jones et al., 2000, y Webb, 2003).

Ahora bien, la discusión teórica respecto a los beneficios y costos de la competencia tributaria no es reciente y se ha desarrollado desde diferentes perspectivas, dos de las cuales son probablemente las más conocidas: la perspectiva económica y la perspectiva política.

Desde la primera, los críticos de la competencia tributaria argumentan que ésta conduce a una subprovisión de bienes públicos, toda vez que provoca una reducción de tasas impositivas de parte de los gobiernos subnacionales, en un intento por atraer las bases impositivas móviles. Es decir, este tipo de competencia, se argumenta, conduce a niveles subóptimos de tasas. Los fundamentos teóricos de esta corriente de pensamiento más representativos se pueden atribuir a Oates (1972), Zodrow y Mieszkowski (1986), Wilson (1986) y Janeba y Peters (1999).

En el extremo opuesto, y producto de trabajos más recientes, se argumenta y se plantean condiciones bajo las cuales la competencia tributaria horizontal induce a una mayor eficiencia (véase, Oates y Schwab, 1988). Esta literatura, que en las dos últimas décadas ha crecido en número, más que cuestionar el modelo básico de Oates (op. cit.), sugiere que, bajo ciertas condiciones, la competencia tributaria, sin reducir la eficiencia recaudatoria, puede inducir a una mayor eficiencia en la aplicación del gasto público y, de esta manera, contribuir a una mayor transparencia y rendición de cuentas. Para una buena síntesis, véase Wilson (1999). 
Desde la perspectiva política se ha desarrollado, paralelamente, una cantidad importante de trabajos en ambas direcciones, argumentando así en favor o en contra de la competencia. En el corazón de estos debates está el tema de la disciplina fiscal, la corrupción y el desperdicio en el gasto público. Para una buena síntesis véase Eggerty y Birch, 2008.

El tema por supuesto es complejo, tanto desde el punto de vista teórico como empírico, ya que puede hablarse de competencia en impuestos con diversos grados de movilidad, como es el caso del trabajo, el capital, el consumo de bienes, etcétera. Más aún, los debates a menudo se traslapan, ya que con frecuencia se habla de competencia entre regiones, y sin duda los efectos son distintos cuando las regiones son países que cuando las regiones son gobiernos subnacionales, pues entre estos últimos no existen fronteras o restricciones a la movilidad. Finalmente, desde el punto de vista empírico la experiencia internacional es de hecho mixta y el debate está lejos de ser concluyente.

En todo caso, para efectos del presente trabajo es importante destacar que en el caso de México prácticamente no se han explorado ni se han estudiado los beneficios potenciales de una mayor competencia tributaria. En el contexto mexicano hasta ahora ha sido difícil diseñar un sistema menos centralizado en la recaudación, porque no ha sido claro quién, aparte del contribuyente, podría salir beneficiado. Los gobiernos estatales, como ya se documentó, han sido los principales beneficiarios y por su parte, al menos hasta hace algunos años, el gobierno federal se ha resistido a esta descentralización de ingreso con argumentos de eficiencia, aunque también es altamente probable que haya resistencia a "perder control" de los estados, como tradicionalmente se concebía. Una hipótesis central en este trabajo es que, a menos de que se logre un cierto grado de descentralización de ingreso, el sistema actual tenderá inevitablemente a mostrar poca responsabilidad fiscal.

\section{Perspectivas}

\section{Cuantificación de la descentralización del gasto}

Lo comentado hasta ahora acerca de la descentralización del gasto requiere de un análisis específico y, en particular, de una medida que permita cuantificar la magnitud del proceso. A este respecto, para una gran diversidad de países existen estimaciones de lo que se ha dado en llamar efecto matamoscas (flypaper effect).

Dentro del análisis del federalismo fiscal, la función desempeñada por las transferencias intergubernamentales se ha convertido en un asunto de gran trascendencia, 
sobre todo en países donde el proceso de descentralización es relativamente reciente. En particular, en el caso de algunos países de América Latina la descentralización del gasto público del gobierno federal a los subnacionales ha tenido importantes efectos macroeconómicos, toda vez que la creciente transferencia del gasto a los gobiernos subnacionales llegó a ser lo suficientemente importante como para influir en las cuentas macroeconómicas de un país. Quizás el mejor ejemplo, aunque no el único, es el de Argentina (véase, Jones et al., 2000).

Sobre este punto, en las últimas décadas una abundante literatura, tanto teórica como empírica, se ha enfocado al impacto que las transferencias intergubernamentales han tenido en las decisiones de los gobiernos que reciben dichas transferencias. Siguiendo a Gamkhar y Oates (1996), en el plano teórico, se ha establecido, por ejemplo, que las transferencias (lump-sum) a una localidad en un esquema de información perfecta deberían tener efectos asignativos y distributivos, no distintos a los que se producirían si la transferencia se hiciera a los residentes de la localidad. Es decir, la respuesta del gasto local de dichas transferencias debería ser equivalente a un aumento del ingreso privado.

Sin embargo, una importante cantidad de literatura empírica ha rechazado este resultado, ya que se observa que el estímulo del gasto a los programas públicos excede, con mucho, a aumentos en el ingreso privado. Dicho de otra forma, la documentación empírica existente muestra que las transferencias a gobiernos subnacionales provocan un sobreestímulo en el gasto local muy superior a incrementos equivalentes en el ingreso privado. El resultado de ello es que el tamaño del gasto público crece por el aumento más que proporcional del gasto público subnacional. Este fenómeno es conocido en la literatura como el flypaper effect o efecto matamoscas, para indicar que el dinero se queda donde cae "money sticks where it hits" (véase Gramlich, 1977; Fisher, 1982; Gamkhar y Oates, 1996).

El resultado tiene implicaciones importantes de política porque sugiere, entre otras cosas, que los gobiernos subnacionales buscan expandir el gasto público para sus propios fines, más allá de los niveles posiblemente deseados por la comunidad. De hecho, una repercusión sería que la competencia política es por sí sola insuficiente para garantizar disciplina fiscal (véase, Oates, 1999). Para el caso de México, sin duda éste es un tema que requiere documentarse, pues, con excepción de Velázquez (2006), no existen trabajos al respecto, ya que la mayoría de los estudios en el país se han orientado al ingreso, no al gasto (véase Ibarra, 1999). Entonces es importante profundizar en el trabajo de los determinantes del gasto estatal, analizando los distintos componentes de las transferencias en los últimos años. 
Una explicación de esta escasez de trabajos es que hasta hace pocos años la información disponible respecto del gasto público transferido a los gobiernos estatales y municipales en México era poco sistemática, pues se requieren datos tipo panel, ${ }^{8}$ desagregándolos por tipo de gasto y localidad (entidad federativa), que con anterioridad a la creación del llamado Ramo 33 no existía. De manera adicional, la presencia de excedentes petroleros, de los cuales una porción importante se ha destinado a los estados, sugiere que un análisis de este tipo es muy pertinente.

La literatura refiere a diversas explicaciones a este fenómeno, desde ilusión fiscal o problemas estadísticos, hasta efectos de sustitución de impuestos. En el caso particular de países en desarrollo, se sugiere también, además de factores económicos, aspectos políticos e institucionales (Tanzi 1996, Prud'homme, 1995).

\section{Conclusiones}

Los cambios operados en las últimas tres décadas en el esquema de relaciones fiscales intergubernamentales han conducido a un esquema en el que el gobierno federal se encarga de la recaudación de la casi totalidad de los ingresos, en tanto que, por el lado del gasto, se ha producido una descentralización importante. De continuar esta tendencia, se pone en riesgo la solvencia misma de las finanzas gubernamentales, ya que en caso de problemas financieros, el gobierno federal es el que tendría que ser el recurso en última instancia. Algunas experiencias internacionales sugieren que éste es un problema latente. Se argumenta entonces que una forma de disminuir este riesgo es involucrando de manera mucho más activa a los gobiernos estatales en la recaudación de impuestos. Ello si bien no elimina la presencia de un gobierno central como garante de última instancia, sí presionaría a los gobiernos subnacionales a no sobrepasar sus límites de gasto ya que, al menos parcialmente, el gasto tendría que estar respaldado por el crecimiento de los ingresos propios.

Más allá de este problema potencial, debe reconocerse que el sistema en su conjunto ha ganado en eficiencia, y la discrecionalidad con la que antes se asignaba el gasto entre entidades prácticamente ha desaparecido, para dar paso a fórmulas o criterios de reparto que, con independencia de su bondad o "justicia", son del conocimiento de todas las partes involucradas. Es decir, se ha ganado transparencia en los criterios de asignación del gasto público del gobierno federal a gobiernos estatales y municipales.

8 La técnica más usual es utilizar modelos “pooled”, entre grupos y mínimos cuadrados generalizados en dos etapas. 
La experiencia en algunos países y la literatura al respecto advierten que un desbalance vertical, como el que se ha producido en los últimos años en México, no favorece la responsabilidad fiscal ni la rendición de cuentas, lo cual es particularmente grave si se tiene en mente que la experiencia en materia de eficiencia del gasto público en México, en las últimas tres décadas, no ha sido muy buena.

Esta situación exige, para inducir algún mayor grado de responsabilidad fiscal al sistema en su conjunto, que los gobiernos subnacionales - y particularmente los estatales- participen de manera más activa en el proceso recaudatorio. Ello implica, si bien de manera parcial y coordinada, que las entidades federativas deberían participar en algún o algunos de los llamados impuestos de base amplia, a fin de que parte de su gasto dependa de su propio esfuerzo recaudatorio y, quizás más importante, que los resultados del gasto estatal estén más vinculados a los contribuyentes de la propia localidad o región. Un cambio de esta naturaleza debería ser acompañado de una modificación simultánea del papel del gobierno federal, a fin de que se fortalezca su responsabilidad de nivelador de desigualdades, tratando con ello de fortalecer la función solidaria del federalismo.

Como se vio, los planteamientos teóricos recientes y la experiencia internacional sugieren que sí existe margen para la competencia tributaria, debidamente acotada y con una adecuada coordinación entre los distintos ámbitos de gobierno.

\section{Bibliografía}

Cabrero, Enrique, La nueva gestión municipal en México, México, Miguel Ángel PorrúaCIDE, México, 1995.

Eggert, W. y P. Sørensen, “The effects of tax competition when politicians create rents to buy political support", en Journal of Public Economics, núm. 92, 2008, pp. 11421163.

Fisher, R., "Income and grant effects of local expenditure: the flypaper effect and other difficulties", en Journal of Urban Economics, núm. 12, 1982, pp. 324-345.

Gamkhar, S. y W. Oates, "Asymmetries in response to increases and decreases in intergovernmental grants: Some empirical findings", en National Tax Journal, núm. 49, 1996, pp. 500-511.
Gil, F. y W. Thirsk, "La prolongada reforma fiscal de México", en Gaceta de Economía, suplemento, "La Política Fiscal en México", año 5, núm. 9, ITAM, México, 2005.

Gordon, R., "An optimal taxation approach to fiscal federalism", en Quarterly Journal of Economics, vol. XCVIII, núm 4, 1993, pp. 567-586.

Gramlich, E., "Intergovernmental grants: A review of the empirical literature", en W. E. Oates (ed.), The Political Economy of Fiscal Federalism, Lexington, 1977, pp. 219-239.

Ibarra, S, J. Sandoval y S. Cervantes, "México: ingresos estatales y dependencia de las participaciones federales", en Comercio Exterior, vol. 49, núm. 5, 1999, pp. 438-444. 
Jones, M., P. Sanguinetti y M. Tomáis, "Politics, institutions and fiscal performance in a federal system: an analysis of the Argentine provinces", en Journal of Development Economics, vol. 61, 2000, pp. 305-333.

Molinar, J. y A. Weldon, "Electoral Determinants and Consequences of Nacional Solidarity, en Wayne A. Cornelius, Transforming State Society Relations in Mexico: The National Solidarity Strategy, San Diego, Center US-Mexican Studies, University of California, 1994.

Oates, W., "An essay on fiscal federalism", en Journal of Economic Literature, núm. 37, 1999, pp. 1120-1149.

, Fiscal federalism, Nueva York, Harcourt Brace Jovanovich, 1972.

,y R. Schwab, "Economic competition among jurisdictions: efficiency enhancing or distortion inducing?", en Journal of Public Economics, núm. 35, 1988, pp. 333354.

Prud'homme, R., "On the dangers of decentralization", en World Bank Research Observer, August, World Bank, 1995.

Rodden, J., G. Eskeland y J. Litvack, "Fiscal Decentralization and the Challenge of Hard Budget Constraints", Londres, MIT Press, 2003.

Sobarzo, H., "Esfuerzo y potencialidad fiscal de los gobiernos estatales en México. Un sistema fiscal representativo", en El Trimestre
Económico, vol. LXXIII(4), México, octubre-diciembre, núm. 292, 2006.

Tanzi, V., "Fiscal federalism and decentralization: A review of some efficiency and macroeconomic aspects", en Annual World Bank Conference on development economics, Washington, DC, World Bank, 1996, pp.295-316.

UCEF, "Diagnóstico integral de la situación actual de las haciendas públicas estatales y municipales, México, SHCP, 2006.

Velázquez, C., "Determinantes del gasto estatal en México", en Gestión y Política Pública, vol. XV, núm. 1, CIDE, 2006, pp. 83-108.

Webb, S., "Fiscal Responsibility Laws for Subnacional Discipline: The Latin American Experience", World Bank, 2003.

Wildasin, D., "Externalities and Bailouts: Hard and Soft Budget Constraints in Intergovermental Fiscal Relations", Working Paper 1883, World Bank, 1997.

Wilson, J., "Theories of Tax Competition", en National Tax Journal, vol. 52, núm. 2, 1999, pp. 269-304.

"A theory of interregional tax competition", en Journal of Urban Economics, núm. 19, 1996, pp.296-315.

Zodrow, G. y P. Mieszkowski, "Pigou, Tiebout, property taxation and the underprovision of local public goods", en Journal of Urban Economics, núm. 19, 1986, pp. 356-370. 